\title{
Intramural aortic haematoma causing ischaemia of the spinal cord
}

\author{
John D Ferguson, Niall Moore, Adrian P Banning
}

\section{Department of Medicine J D Ferguson Department of Radiology N Moore \\ Department of Cardiology, John Radcliffe Hospital, Oxford \\ A P Banning \\ Correspondence to: Dr A P Banning, Department of Cardiology, John Radcliffe Hospital, Oxford OX3 9DU. \\ Accepted for publication 7 November 1995}

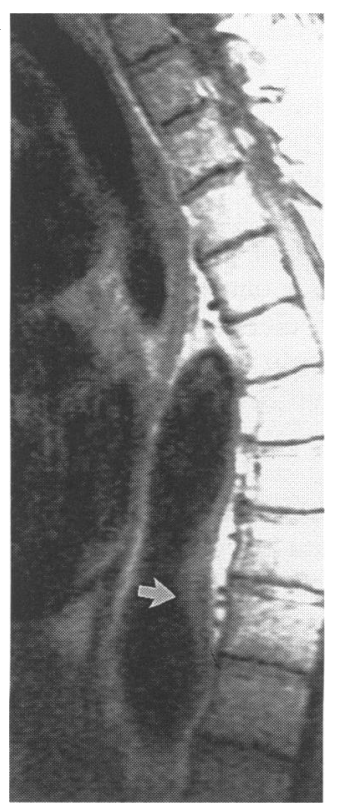

Figure 1 Sagittal T1 weighted magnetic resonance image of the descending aorta. There is a crescentic area of aortic wall thickening (arrow) with no evidence of an intimal flap or a dissection membrane.
A 62 year old man with a history of hypertension presented with sharp intrascapular back pain of sudden onset. The pain was accompanied by a progressive loss of sensation extending up to the level of the xiphisternum and symmetrical loss of lower limb power. Over the ensuing 90 minutes, his symptoms largely resolved apart from residual back pain and leg parasthesiae.

Examination revealed a blood pressure of $140 / 75 \mathrm{~mm} \mathrm{Hg}$, no peripheral pulse deficits, and no evidence of aortic regurgitation. Tendon reflexes at the knee and ankle were depressed and plantar reflexes were equivocal. Sensation by this time was normal.

A chest radiograph showed a dilated ascending aorta but no evidence of aortic dissection. Radiographs of the spine, electrocardiogram, and routine biochemical and haematological investigations were unremarkable. Magnetic resonance imaging and computed tomography showed a normal spinal cord and ascending aorta but revealed two discrete areas of wall thickening in the descending aorta: one $0.8 \times 6$ $\mathrm{cm}$ in length, distal to the left subclavian artery, and a similar abnormality $0.5 \times 5 \mathrm{~cm}$ in length at the level of D9 (figs 1 and 2). Transoesophageal echocardiography confirmed the normal ascending aorta and the abnormality in the upper descending aorta; these were diagnosed as intramural aortic haematomas. The patient's pain resolved rapidly and following aggressive control of the blood pressure he was discharged fit and well with no neurological abnormality.

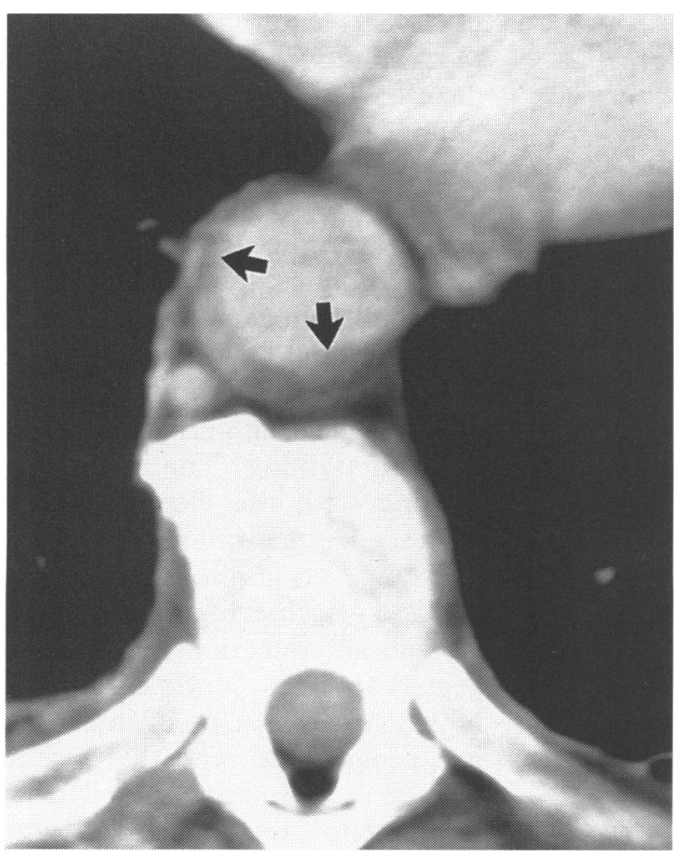

Figure 2 Transverse computed tomographic section at the level of D9. The area of aortic wall thickening is marked (arrow).
Three weeks later he developed further back pain and an acutely ischaemic right leg. Examination revealed signs of aortic regurgitation, and computed tomography showed an extensive type A aortic dissection. At thoracotomy there was a transverse tear anteriorly in the mid ascending aorta. Excision of the intimal tear and replacement of the ascending aorta was performed without complication and the patient was discharged 10 days later.

This patient presented with symptoms and signs of spinal cord ischaemia. Investigation showed two discrete areas of abnormality in the aorta which were diagnosed as intramural haematomas. The principal blood supply of the thoracic spinal cord is the anteria radicularis magna, which usually arises from the aorta at the level of D10. This corresponds to the level of the haematoma in the descending aorta in this patient. We postulate that disruption of the aortic wall compromised the spinal cord blood supply, resulting in paralysis. Resolution of the symptoms may have corresponded to either the development of a collateral blood supply or to decompression of occlusive haematoma around the arterial origin.

Intramural haematoma is a distinct pathological entity representing spontaneous localised haemorrhage into the aortic wall. ${ }^{1}$ The clinical presentation often mimics that of dissection, and the diagnosis can only be made when an intimal tear has been excluded. Magnetic resonance imaging and transoesophageal echocardiography typically show circular or crescentic thickening of the aortic wall $>0.7 \mathrm{~cm}$ depth and $<20 \mathrm{~cm}$ in length, associated with central displacement of any intimal calcification. ${ }^{2}$

Intramural haematoma of the descending aorta preceding dissection of the ascending aorta has been described before. ${ }^{1}$ The mechanism is unclear, but the presence of the haematoma may reflect a generalised aortic abnormality and a predisposition to dissection. Up to $50 \%$ of patients with intramural haematoma will subsequently develop dissection or aortic rupture, despite aggressive blood pressure control. Some centres regard an intramural haematoma in the ascending aorta as an indication for surgery. ${ }^{1-3}$ We believe this is the first case of aortic haematoma presenting with transient occlusion of the spinal artery.

We are grateful to Dr JM Holt for allowing us to report this case.

1 Robbins RC, McManus RP, Mitchell RS, et al. Management of patients with intramural haematoma of the thoracic aorta. Circulation 1993;88:1-10.

2 Mohr-Kahaly S, Erbel R, Kearney P, Puth M, Meyer J. Aortic intramural hemorrhage visualised by transesophageal echocardiography: findings and prognostic implications. $\mathcal{F}$ Am Coll Cardiol 1994;23:658-6.

3 Neinaber CA, von Kodolitsch Y, Peterson B, et al. Intramural hemorrhage of the thoracic aorta; diagnostic and therapeutic implications. Circulation 1995;92:1465-72. 